\title{
Marine Reserve Creation and Interactions between Fisheries and Aquaculture - A Bio-economic Model Analysis
}

\author{
Bui Bich Xuan \& Claire W. Armstrong \\ Norwegian College of Fishery Science, University of Tromsø The Arctic University of Norway, 9037 \\ Tromsø, Norway
}

\begin{abstract}
The rapid growth of aquaculture world-wide affects capture fisheries in several ways. We present a bio-economic model to clarify interactions between these two activities, including the effect of a marine protected area (MPA). The aquaculture production is assumed to reduce the intrinsic growth rate of fish, and hence stock size and harvest, while the dispersal from the MPA may limit these effects. This is considered applying both open access and economically optimal management outside the MPA. The results of a numerical simulation show that the wild fish stock in an open access fishery is independent of the aquaculture development, while the wild catch significantly declines. Introducing an MPA, the wild fish stock increases, while the wild catch varies depending on the aquaculture development and the MPA size. With optimal management, the stock size declines for small MPA sizes, but increases for larger sizes, while aquaculture production declines for large MPA size. The harvest and the total profits increase with increasing MPA size. These results show that an MPA may not only compensate for negative effects of aquaculture upon a wild fishery, but may be biologically and economically enhancing under both open access and optimal management regimes as well.
\end{abstract}

JEL Codes: Q22, Q28

KEYWORDS: Wild fishery, aquaculture, marine protected areas, bioeconomic model, management

\section{Introduction}

Total world fish production increased dramatically from 19.3 million tons in 1950 to 148.5 million tons in 2010, with the largest contribution coming from marine capture fisheries (FAO, 2012). However, fish supply from the wild capture fisheries reached a peak and has been fairly stable at about 90 million tons since the late 1980s. Since then, the increase in total fish production has come from aquaculture (Diana, 2009). Aquaculture production reached 59 million tons in 2010, compared with 4.7 million tons in 1980, which is equivalent to an average annual rate of increase of 38.5 percent (FAO, 2013). This rapid growth has many effects, and we will in this paper apply a bioeconomic model to study the interactions between a wild fishery and aquaculture. The focus will be on negative externalities of aquaculture on the fishery, and how the implementation of marine protected area might alleviate these effects. Aquaculture development does not only give a substantial and growing contribution to world fish supply, but also provides positive benefits to national and regional economies, poverty reduction and food security (Fisheries, FAO, 2011). In addition, aquaculture growth has been suggested to dampen the overexploitation in the commercial capture fisheries. Both Anderson (1985) and Ye and Beddington (1996) have analyzed market interactions between fisheries and aquaculture. They show that the presence of aquaculture reduces fishing effort in commercial 
fisheries and lowers the fish price. This leads to increased wild fish stock and catch. Asche and Tveterås (2004) carried out research on the relationship between aquaculture and declining fisheries. They show that the increase in aquaculture has not led to more pressure in the fisheries for fishmeal, unless the fisheries are open access and there are no close substitute products for fishmeal.

However, there are also many studies criticizing the growth of aquaculture due to negative effects on capture fisheries both directly and indirectly. Hannesson (2003) used a two-species system of feed fish and edible fish to discuss the interrelationship between aquaculture and fisheries. He shows that the growth of aquaculture does not improve the overfishing problem under open access but ultimately leads to overexploitation of feed fish. This has an adverse effect on edible fish stocks due to food reduction for edible fish and predators in the sea, finally leading to diminished catch of edible fish. Tacon and Metian (2009) statistically analyzed the aquaculture production systems in many Asian countries, which are mostly based on the use of low value/trash fish species as feed inputs. They show that aquaculture has increased fishing pressure on local fish resources. Naylor et al. (2000) also point to the fact that some aquaculture systems reduce wild fish supplies through exploitation of wild seed and trash fish for culturing. Moreover, there are other ecological impacts from the growth of aquaculture such as environmental damage, water and genetic pollution, destruction of mangrove forests, and the spread of disease from the cultured fish to wild fish (Chopin et al., 2001; Naylor et al., 2000). These are important aspects when exploring the implications of coexistence of commercial fisheries and aquaculture in particular ocean regions. Hoagland et al. (2003) presented a bio-economic model in which aquaculture activities reduce the environmental carrying capacity of the fish stock and thus the resulting stock size and harvest from wild fishery. They emphasize that the "effective" carrying capacity of wild fish may be reduced due to competition in relation to ocean space or as a result of juvenile harvests from wild stocks to supply aquaculture operations. An example of this is the commercial lobster fishery and lobster aquaculture in Vietnam, which shows that the presence of the later has put pressure on this unregulated fishery, largely resulting in its disappearance. Since the 1990s, the size of wild lobster caught has continued to decrease to a size smaller than that which is demanded by the export market. This has led to fishermen holding lobsters in simple net cages and feeding them up to bigger and more valuable size. As this practice has been recognized to be very profitable, more and more effort has been put into catching wild lobster juveniles for aquaculture. This has therefore caused over-exploitation and disappearance of the commercial non-juvenile lobster fishery since the 2000s (Thuy \& Ngoc, 2004). The number of juveniles caught annually in Khanh Hoa province, where the culture of lobsters is the most productive, declined from 600 thousand individuals in 2005-2007 to 200 thousand individuals in 2008-2012. The average size of juveniles caught decreased from $9.5 \mathrm{~mm}$ in 2003 to $7.2 \mathrm{~mm}$ in 2013 (Phu et al., 2013) 
Though many economic studies have been critical of the effect of marine protected areas (MPAs) on fisheries (Hannesson, 1998; Smith \& Wilen, 2003), MPAs may soften the negative externalities effects of aquaculture. The use of MPAs as a fisheries management tool has been widely studied in recent years. In an early work on a marine sanctuary and fishing grounds system, Hannesson (1998) showed that the catch in adjacent exploited areas might be greater than under open access due to migration to fishable areas from a more plentiful stock in the MPA. But he underlines that reserves give little or no benefit without effective control of effort or catches. Armstrong and Reithe (2001) added a management cost function to Hannesson's model and increased the fishing costs to a realistic level in order to show that a certain size of an MPA can be a better management tool than traditional quotas. A marine reserve can increase resource rents to fishers and reduce the recovery time for the harvested population in the presence of negative shocks (Grafton et al., 2006, 2009). In a spatial bioeconomic model, both total fish biomass and harvest may increase after closing and protecting an area from exploitation (Sanchirico \& Wilen, 2001).

Our study is based on Hoagland et al. (2003). However, we assume that aquaculture affects the intrinsic growth rate of the fish stock, rather than the carrying capacity. Simon et al. (2012) carried out a study of the relationship between the key life history parameters (e.g., pre-recruit mortality rate, post-recruitment mortality rate, and annual fecundity) and the intrinsic growth rate in a dynamic biomass model. They show that the natural mortality of pre-recruit stages (larvae and juveniles) is the parameter most strongly affecting the intrinsic population growth rate. The natural mortality of the early life stages takes into account both density-dependent and density-independent mortality rate, such as death by predation, competition, starvation, disease, or hostile environment conditions. If we consider catching juveniles from a wild stock to supply aquaculture, as in the Vietnamese lobster case, such an operation increases the natural mortality of the wild stock in the early stages. Hence, catching juveniles may be expected to reduce the intrinsic growth rate. This, again, reduces the natural growth rate of the wild stock. Catching juveniles from a wild stock to supply for aquaculture operation is typical in the culture of bluefin tuna, Atlantic sea scallops, summer flounder (Hoagland et al., 2003), and spiny lobster (Petersen et al., 2011).

Hence our focus is aquaculture's effect on the wild stock's intrinsic growth, as well as including the effects of introducing an MPA. We present two models, one with and one without an MPA, in order to explore the possible effects of an MPA on the interactions between aquaculture and the wild fishery. The first model is set up for a system of aquaculture-fishery interactions in a coastal area, where we assume that aquaculture has an external effect reducing the intrinsic growth rate of the fish stock. In the second model, an MPA is established in a portion of the coastal area, which is protected from fishing and aquaculture, while the remaining portion is left for both users of the ocean. Four management regimes are studied: pure open access, open access to the area outside the MPA, 
economic optimum with and without an MPA. Unfortunately, due to the complexity of the interactions, analytical results are not forthcoming; hence numerical simulation is conducted in order to investigate the behavior of the models.

Questions analyzed in our study are: What is the trade-off between a wild fishery and aquaculture? Can introducing an MPA reduce the negative effects of aquaculture on a wild fishery (both in stock and harvest) in the open access case? What is the economically optimal outcome from coexistence of marine farmers and fishermen with and without the MPA effects? This study makes several contributions to the literature. The first is expanding Hoagland et al's (2003) model in order to show the effects of the introduction of an MPA on the interactions between a wild fishery and aquaculture. Secondly, we apply the habitat effect to the intrinsic growth, and finally, we present MPAs as an integrated management tool to reduce potential conflicts between different users of the sea, in the presence of an open access fishery.

The numerical results show that under pure open access, the equilibrium wild catch decreases with aquaculture development, while the stock size is not affected by aquaculture. Given the amount of aquaculture activity, introducing an MPA increases the total stock of wild fish, while the wild catch varies depending on the MPA size. The wild catch either increases and reaches the maximum value at a certain MPA size or decreases and is lower than when there is no MPA. A comparison between the optimized systems of a wild fishery and aquaculture with and without an MPA shows that creation of an MPA of a certain size results in higher optimal values (i.e., the total wild stock size, wild catch, aquaculture production, and total net profits), compared to when there is no MPA.

The remainder of the paper is organized as follows. The next section describes our models. Section three presents the two management regimes. In section four, we simulate our approach with a numerical example. Section five summarizes the major conclusions and suggests some additional future research.

\section{Models}

\section{Basic fishery model}

We start with the original situation where the entire area is open for fisheries. Suppose the fish stock obeys the logistic law of growth described by the net growth equation

$$
\dot{X}=F(X)-Y_{f}=r X(1-X)-Y_{f}, \quad X=\frac{x}{K}
$$

where $F(X)$ is a strictly concave natural growth function, $X$ denotes the fish stock measured as density, $r$ denotes the intrinsic growth rate, $x$ is the stock size, $K$ denotes the carrying capacity of the fish stock, and $Y_{f} \geq 0$ represents the harvest rate of fish expressed as density. 
The net benefit function is described as:

$$
\pi\left(X, Y_{f}\right)=\left(p-\frac{c}{q X}\right) Y_{f}
$$

where, $c$ is a constant unit cost of fishing effort, $q$ denotes catchability, and $p$ is constant unit price of fish. All parameters are positive.

\section{External effect from aquaculture on the wild fishery}

Now let us introduce aquaculture that coexists with fisheries on the fishing grounds. The presence of aquaculture will decrease the intrinsic growth rate of the fish stock (Simon et al., 2012). Let $N$ be the total measure of area or volume devoted to aquaculture, and let $r$ be a linear function of $N$ so that the intrinsic growth rate changes with $N$ relative to a positive aquaculture sensitivity coefficient, $\alpha$ :

$$
r(N)=r-\alpha N>0, \quad \text { for all } \mathrm{N}
$$

The net growth equation will now be

$$
\dot{X}=F(X, N)-Y_{f}=r(N) X(1-X)-Y_{f}
$$

The economic dimensions of the culturing operation will be characterized following Hoagland et al. (2003). We specify a linear production function for aquaculture taking the form: $Y_{a}(N)=w N$, where $Y_{a}(N)$ is the total farmgate output, $w$ is the positive and constant output per aquaculture area. The larger the amount of fish production supplied to the market, the more aquaculture area, $N$, is needed. We assume that the operating cost of aquaculture (e.g., feed, seeds, labor, boats, interest, and other capital items), $C_{a}(N)$, is a linear function of the total aquaculture area, in order to simplify the analysis: $C_{a}(N)=v N$. The cost of investment in new aquaculture facilities (e.g., the components and devices of aquaculture), $I(z)$, is a linear function of the increment, $z$, to the total aquaculture area $N$ : $I(z)=b z$. Where $v$ and $b$ are positive and constant parameters denoting the unit operating cost of aquaculture and the cost of investment in a new aquaculture facilities, respectively.

We assume wild fish and cultured fish have the same market price, $p$. The total net benefit is defined as the sum of net benefits from the wild fishery and aquaculture taking the form:

$$
V(X, N)=\left[\left(p-\frac{c}{q X}\right) Y_{f}\right]+\left[p Y_{a}(N)-C_{a}(N)-I(z)\right]
$$

\section{MPA creation}

Now let us include an MPA in the system: sub-area 1 is set up for both the wild fishery and aquaculture and sub-area 2 is set up for marine reserve purposes. Fishing and aquaculture in the MPA is forbidden. The total population, hence, is divided into two sub-populations, $x_{1}$ and $x_{2}$, with the 
same homogeneous characteristics, and $x=x_{1}+x_{2}$. Two sub-stock densities are defined as $X_{1}$ and $X_{2}$, respectively, and $X=X_{1}+X_{2}$, where $X_{1}=\frac{x_{1}}{K}$ and $X_{2}=\frac{x_{2}}{K}$. The total distribution area is set equal to unity and sub-areas 1 and 2 equal $(1-m)$ and $m$, respectively, $0<m<1$. We assume that each sub-population has its own carrying capacity and that it is proportional to the size of the sub-area. The creation of an MPA raises the possibility of migration or diffusion if there is a difference between subpopulation densities, i.e., $\frac{X_{1}}{1-m}=\frac{x_{1}}{(1-m) K}$ and $\frac{X_{2}}{m}=\frac{x_{2}}{m K}$. We assume that net migration moves from the MPA, where there is higher population density compared to the fishing ground. Therefore, net emigration from the MPA equals immigration to the fishing ground at a rate $M\left(X_{1}, X_{2}\right)=$ $\gamma\left(\frac{X_{2}}{m}-\frac{X_{1}}{1-m}\right)$, where $\gamma>0$ is the migration coefficient. ${ }^{1}$ To keep things simple, we assume that $r_{1}=r_{2}$ $=r$, and the intrinsic growth rate of fish stock in the fishable area follows (3). The sub-population net growths in the two areas are:

$$
\begin{aligned}
\dot{X}_{1} & =F_{1}\left(X_{1}, N\right)+M\left(X_{1}, X_{2}\right)-Y_{1 f} \\
& =(r-\alpha N) X_{1}\left(1-\frac{X_{1}}{(1-m)}\right)+\gamma\left(\frac{X_{2}}{m}-\frac{X_{1}}{(1-m)}\right)-Y_{1 f} \\
\dot{X}_{2} & =F_{2}\left(X_{2}\right)-M\left(X_{1}, X_{2}\right)=r X_{2}\left(1-\frac{X_{2}}{m}\right)-\gamma\left(\frac{X_{2}}{m}-\frac{X_{1}}{(1-m)}\right)
\end{aligned}
$$

where, $Y_{1 f}$ is the catch rate in sub-area 1 , expressed as density.

The total net benefits from the wild fishery and aquaculture take the form:

$$
V\left(X_{1}, N\right)=\left[\left(p-\frac{c}{q X_{1}}\right) Y_{1 f}\right]+\left[p Y_{a}(N)-C_{a}(N)-I(z)\right]
$$

\section{Management regimes}

\section{An Open Access Regime}

\section{External effect from aquaculture on the wild fishery}

The external effect from aquaculture on the wild fishery depends on the amount of aquaculture $(N)$. We assume that aquaculture is exogenously determined in the open access fishery case. In open access equilibrium in the fishery, the stock density is $X^{\infty}=\frac{x^{\infty}}{K}=\frac{c}{K q p}$ and the harvest rate is derived from equation (4) giving $Y_{f}^{\infty}=F\left(X^{\infty}, N\right){ }^{2}$ The equilibrium wild stock is independent with the presence of

\footnotetext{
${ }^{1}$ Papers studying bieconomic effects of MPAs often assume that each sub-population has its own carrying capacity, e.g., Hannesson (1998); Conrad (1999); Flaaten and Mjølhus (2010).

${ }^{2}$ The net profit of the fishery is $\pi(x, y)=\left(p-\frac{c}{q x}\right) y$, where $y$ is harvest rate in tons. In open access the net profit becomes to zero, and the stock size will be $x^{\infty}=\frac{c}{q p}$, while the stock density is $X^{\infty}=\frac{x^{\infty}}{K}=\frac{c}{q p K}$.
} 
aquaculture while the equilibrium wild catch dependent on aquaculture production. The wild catch goes down when the amount of aquaculture goes up.

\section{MPA creation}

When we introduce an MPA in the system, the grounds available for fishing and aquaculture become smaller than without an MPA. In the equilibrium, the stock density in the MPA can be calculated as:

$$
X_{2}=-\frac{1}{2}\left(\frac{\gamma}{r}-m\right)+\sqrt{\frac{1}{4}\left(\frac{\gamma}{r}-m\right)^{2}+\frac{\gamma m X_{1}}{r(1-m)}}
$$

For open access in sub-area 1 , the stock density is $X_{1}^{\infty}=X^{\infty}(1-m)=\frac{c}{K q p}(1-m)$. Substituting $X_{1}$ with this in equation (9), we can find $X_{2}$. The wild stock size in the entire area will be $x=x_{1}+x_{2}=$ $K X_{1}^{\infty}+K X_{2}$. From equation (6) and (7) we can find the equilibrium catch rate $Y_{1 f}^{\infty}=F_{1}\left(X_{1}^{\infty}, N\right)+$ $F_{2}\left(X_{2}\right)$. The catch in tons is $y_{1 f}^{\infty}=(1-m) K\left[F_{1}\left(X_{1}^{\infty}, N\right)+F_{2}\left(X_{2}\right)\right]$.

\section{An Optimal Management Regime}

External effect from aquaculture on the wild fishery

A manager chooses the level of harvest, $Y_{f}$, and the level of investment in aquaculture, $z$, in order to maximize the net present value of fish production from both wild fishery and aquaculture:

$$
\max \int_{0}^{\infty}\left\{\left[\left(p-\frac{c}{q X}\right) Y_{f}\right]+\left[p Y_{a}(N)-C_{a}(N)-I(z)\right]\right\} e^{-\delta t} d t
$$

subject to:

$$
\begin{array}{ll}
\dot{X}=F(X, N)-Y_{f}, & X(0)=X_{o}, \quad X(t) \geq 0, \quad 0 \leq Y_{f} \leq Y_{\text {fmax }} \\
\dot{N}=z, & N(0)=0, \quad N(t) \geq 0, \quad z \geq 0
\end{array}
$$

The current-value Hamiltonian for this problem may be written as:

$$
H^{c}=\left[\left(p-\frac{c}{q X}\right) Y_{f}\right]+\left[p Y_{a}(N)-C_{a}(N)-I(z)\right]+\lambda\left[F(X, N)-Y_{f}\right]+\beta z
$$

With $\lambda$ and $\beta$ being the adjoint variables measuring the shadow prices of the associated state variables $X$ and $N$, the first order conditions for an interior solution include:

$$
\begin{aligned}
& \frac{\partial H^{c}}{\partial Y_{f}}=\left(p-\frac{c}{q X}\right)-\lambda=0 \\
& \frac{\partial H^{c}}{\partial z}=-b+\beta=0
\end{aligned}
$$




$$
\begin{aligned}
& \dot{\lambda}-\delta \lambda=-\frac{\partial H^{c}}{\partial X}=-\frac{c Y_{f}}{q X^{2}}-\lambda r(1-\alpha N)(1-2 X) \\
& \dot{\beta}-\delta \beta=-\frac{\partial H^{c}}{\partial N}=-p w+v+\lambda \alpha X(1-X)
\end{aligned}
$$

Equations (14) through (17) become:

$$
\begin{aligned}
& \lambda=p-\frac{c}{q X} \\
& \beta=b \\
& \dot{\lambda}-\lambda(\delta-(r-\alpha N)+2(r-\alpha N) X)+\frac{c Y_{f}}{q X^{2}}=0 \\
& \dot{\beta}-\delta \beta+p w-v-\lambda \alpha X(1-X)=0
\end{aligned}
$$

In this problem, we have a system of six equations, (11), (12), and (18) through (21), and six unknown variables, $\lambda, \beta, Y_{f}, z, X$, and $N$. The steady-state values for these variables may be solved simultaneously and the present value of the wild fishery and aquaculture at bioeconomic optimum becomes $V\left(X^{*}, N^{*}\right)=\left[\left(p-\frac{c}{q X^{*}}\right) F\left(X^{*}, N^{*}\right)+\left(p w N^{*}-v N^{*}-b z^{*}\right)\right] / \delta^{3}$

\section{MPA creation}

When the original ground is divided into two sub-areas, one is set up for an MPA and the other is used for fisheries and aquaculture. We consider the problem where a manager seeks to maximize the net profits from both fishery and aquaculture:

$$
\max \int_{0}^{\infty}\left\{\left[\left(p-\frac{c}{q X_{1}}\right) Y_{1 f}\right]+\left[p Y_{a}(N)-C_{a}(N)-I(z)\right]\right\} e^{-\delta t} d t
$$

subject to:

$$
\begin{array}{lc}
\dot{X}_{1}=F_{1}\left(X_{1}, N\right)+M\left(X_{1}, X_{2}\right)-Y_{1 f}, & X_{1}(0)=X_{o 1}, X_{1}(t) \geq 0,0 \leq Y_{1 f} \leq Y_{1 f m a x} \\
\dot{X}_{2}=F_{2}\left(X_{2}\right)-M\left(X_{1}, X_{2}\right), & X_{2}(0)=X_{o 2}, X_{2}(t) \geq 0 \\
\dot{N}=z, & N(0)=0, \quad N(t) \geq 0, \quad z(t) \geq 0
\end{array}
$$

The current-value Hamiltonian for this problem may be written as:

$$
H^{c}=\left[\left(p-\frac{c}{q X_{1}}\right) Y_{1 f}\right]+\left[p Y_{a}(N)-C_{a}(N)-I(Z)\right]+\lambda_{1}\left[F_{1}\left(X_{1}, N\right)+M\left(X_{1}, X_{2}\right)-Y_{1 f}\right]
$$

\footnotetext{
${ }^{3} z^{*}$ can be calculated based on $N^{*}$. We define $z$ as an increment in aquaculture activity to the total amount of aquaculture $N$. In equilibrium, $\dot{N}=z=0$, therefore, $z^{*}=0$. The total amount of aquaculture activity $N$ will be equal to the sum of increment $z$ from the initial time to equilibrium: $N^{*}=z^{*}=\sum_{t=0}^{\infty} z_{t}$.
} 


$$
+\lambda_{2}\left[F_{2}\left(X_{2}\right)-M\left(X_{1}, X_{2}\right)\right]+\beta z
$$

With $\lambda_{1}, \lambda_{2}$ and $\beta$ being the adjoint variables measuring the shadow prices of the associated state variables $X_{1}, X_{2}$, and $N$, the first order conditions for an interior solution include:

$$
\begin{aligned}
& \frac{\partial H^{c}}{\partial Y_{1 f}}=\left(p-\frac{c}{q X_{1}}\right)-\lambda_{1}=0 \\
& \frac{\partial H^{c}}{\partial z}=-b+\beta=0 \\
& \dot{\lambda}_{1}-\delta \lambda_{1}=-\frac{\partial H^{c}}{\partial X_{1}}=-\frac{c Y_{1 f}}{q X_{1}^{2}}-\lambda_{1}\left((r-\alpha N)-\frac{2(r-\alpha N) X_{1}}{1-m}-\frac{\gamma}{1-m}\right)-\frac{\lambda_{2} \gamma}{1-m} \\
& \dot{\lambda}_{2}-\delta \lambda_{2}=-\frac{\partial H^{c}}{\partial X_{2}}=-\frac{\lambda_{1} \gamma}{m}-\lambda_{2}\left(r-\frac{2 r X_{2}}{m}-\frac{\gamma}{m}\right) \\
& \dot{\beta}-\delta \beta=-\frac{\partial H^{c}}{\partial N}=-p w+v+\lambda_{1} \alpha X_{1}\left(1-\frac{X_{1}}{1-m}\right)
\end{aligned}
$$

Equations (27) through (31) become:

$$
\begin{aligned}
& \lambda_{1}=\left(p-\frac{c}{q X_{1}}\right) \\
& \beta=b \\
& \dot{\lambda}_{1}-\lambda_{1}\left(\delta-(r-\alpha N)+\frac{2(r-\alpha N) X_{1}}{1-m}+\frac{\gamma}{1-m}\right)+\frac{c Y_{1 f}}{q X_{1}^{2}}+\frac{\lambda_{2} \gamma}{1-m}=0 \\
& \dot{\lambda}_{2}-\lambda_{2}\left(\delta-r+\frac{2 r X_{2}}{m}+\frac{\gamma}{m}\right)+\frac{\lambda_{1} \gamma}{m}=0 \\
& \dot{\beta}-\delta \beta+p w-v-\lambda_{1} \alpha X_{1}\left(1-\frac{X_{1}}{1-m}\right)=0
\end{aligned}
$$

In this problem, we have a system of eight equations, (23) through (25), and (32) through (36), and eight unknown variables, $\lambda_{1}, \lambda_{2}, \beta, Y_{f}, z, X_{1}, X_{2}$ and $N$. The steady-state values for these variables may be solved for simultaneously, and the present value of the wild fishery and aquaculture at bioeconomic optimum becomes $V\left(X_{1}^{*}, N^{*}\right)=\left[\left(p-\frac{c}{q X_{1}^{*}}\right)\left[F_{1}\left(X_{1}^{*}, N^{*}\right)+F_{2}\left(X_{2}^{*}\right)\right]+\left(p w N^{*}-v N^{*}-b z^{*}\right)\right] / \delta$. Because optimal management models with and without an MPA are nonlinear, the analytical solutions involve multiple roots, are lengthy and do not yield any significant insights. Hence, we adopt a numerical procedure that is both relatively simple and gives us more control in searching for interior solutions.

\section{Numerical Simulations}


We now illustrate the open access and optimal management case at equilibrium numerically. We specify the carrying capacity equals to unity and the other parameter values as presented in Table 1.

Table 1. Parameters for Wild Fishery and Aquaculture

\begin{tabular}{|c|l|c|r|}
\hline Variable & \multicolumn{1}{|c|}{ Description } & Unit & Value \\
\hline$w$ & Aquaculture production output per farm & volume/area & 1 \\
$\alpha$ & Aquaculture sensitivity coefficient & (area x time) $^{-1}$ & 100 \\
$v$ & Aquaculture production operating cost & currency/year/area & 0.4 \\
$c$ & Unit cost of fishing effort & currency/day $^{-1}$ & 0.3 \\
$q$ & Catchability coefficient & day $^{-1}$ & 1 \\
$p$ & Unit price of fish & currency/volume $^{-1}$ & 1 \\
$r$ & Intrinsic growth rate & time $^{-1}$ & 0.4 \\
$b$ & Investment cost & & 0.2 \\
$\gamma$ & Migration coefficient & & 0.5 \\
$\delta$ & Discount rate & 0.07 \\
\hline
\end{tabular}

\section{Open access regime}

Figure 1 shows the impact on total stock of varying the MPA size in the case of open access outside the MPA, compared to pure open access. We observe that the stock size is greater when an MPA is introduced. The impact on harvests, as shown in Figure 2, is more diverse, and varies for the amount of aquaculture activity introduced. First of all, the pure open access harvest declines for increased $N$, while the effects in the presence of an MPA are more varied. For example, for $N=0.003$, the wild catch increases and reaches a maximum value for MPA size $m=0.4$. For a large MPA (about $75 \%$ of the entire area), the equilibrium wild catch is the same as without an MPA, while a larger MPA gives lower catch than pure open access. For $N=0.004$, the equilibrium wild catch in pure open access equals to zero, while the wild catch with an MPA increases and reaches a maximum level for MPA $m$ $=0.5$, the larger MPA sizes give a decrease in the wild catch and dissipation when the entire area is set up for an MPA. For $N \leq 0.002$, the wild catch with an MPA declines for an increasing MPA, and is always smaller than the equilibrium wild catch without an MPA. 


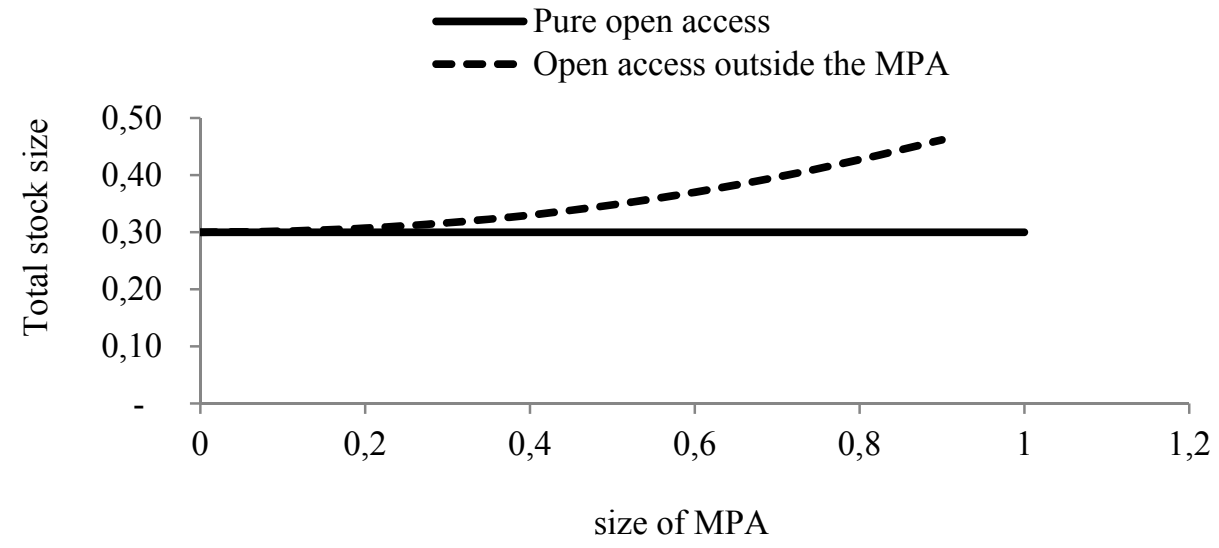

Figure 1. Effect of varying size of MPA on wild fish stock size, under open access.

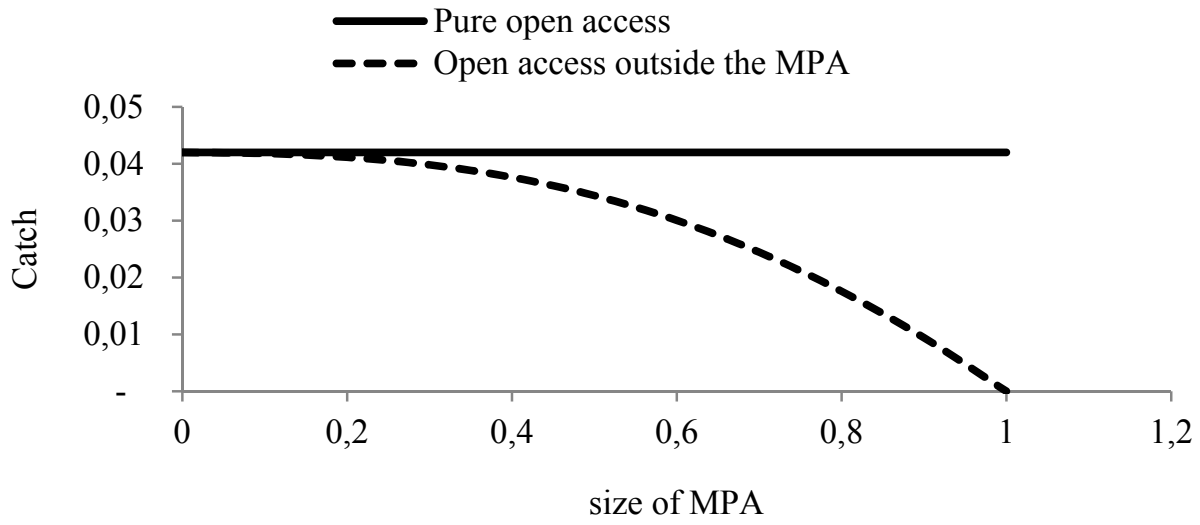

(a) $N=0.002$

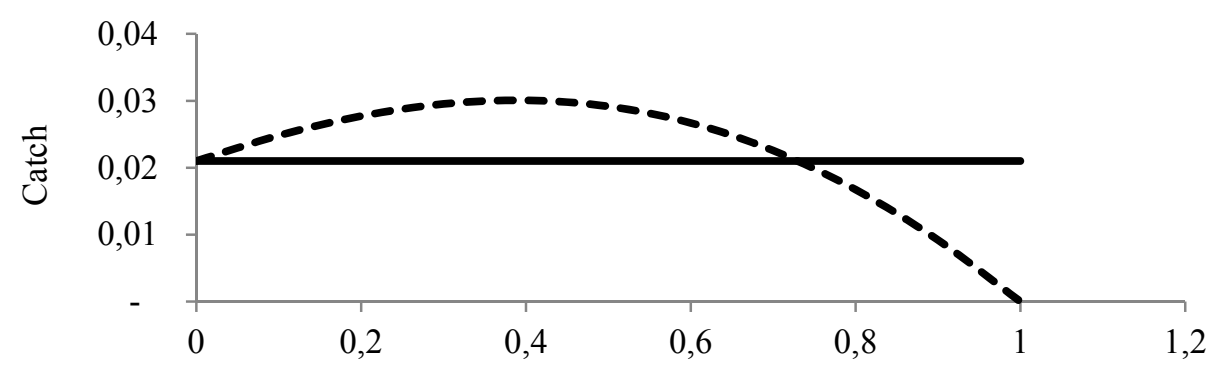

size of MPA

(b) $N=0.003$

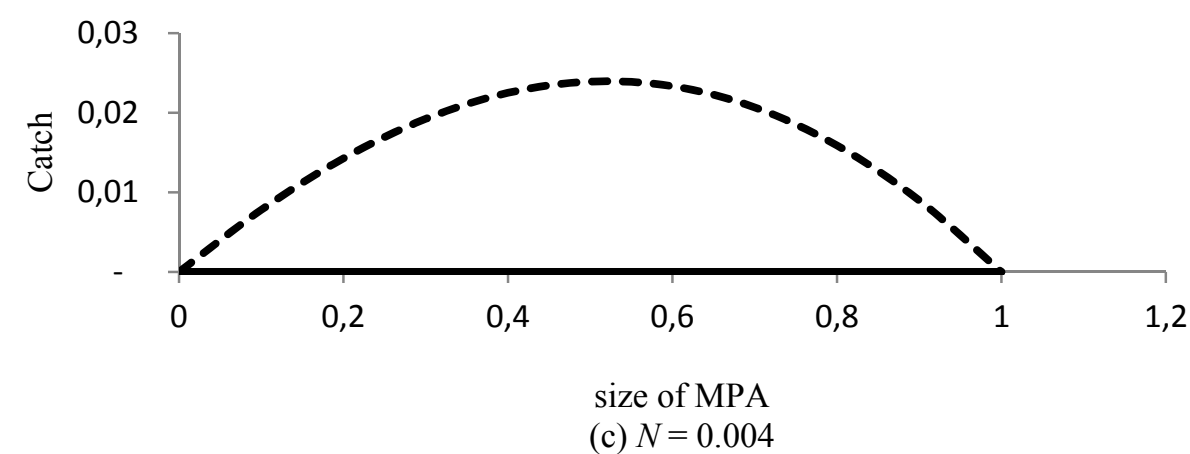


Figure 2. Effect of varying size of MPA on catches given the amount of aquaculture activity a) $N=0.002$, b) $N=0.003$ and c) $N=0.004$, respectively.

\section{Optimal management regime}

When we run the economic optimal model with our specified parameter values, we find the optimal stock levels and harvest rate in densities, the amount of aquaculture, and net profits with and without an MPA. We then calculate stock sizes and harvest rate in volume as in Table 2.

Table 2. Simulation results for the steady-state equilibria in the bioeconomic optimum ${ }^{4}$ (all the values in $10^{-3}$ )

\begin{tabular}{|c|l|c|r|r|r|r|r|}
\hline Output & \multicolumn{1}{|c|}{ Description } & Unit & Without & \multicolumn{3}{|c|}{ With MPA $(m)$} \\
\cline { 5 - 7 } variable & & & MPA & 0.1 & 0.3 & \multicolumn{1}{c|}{0.5} & \multicolumn{1}{c|}{0.6} \\
\hline$x_{1}^{*}$ & Stock size in fishing ground & volume & & 278 & 217 & 158 & 135 \\
$x_{2}^{*}$ & Stock size in MPA & volume & & 4 & 45 & 178 & 321 \\
$x^{*}$ & Total stock size & volume & 309 & 282 & 262 & 336 & 456 \\
$N^{*}$ & Amount of aquaculture activity & area & 4.0 & 4.4 & 5.7 & 7.3 & 5.6 \\
$y_{f}^{*}, y_{1 f}^{*}$ & Harvest in tons & volume & 0.6 & 0.7 & 0.9 & 1.5 & 5.9 \\
$V^{*}$ & Total net profits & currency & 1.6 & 1.8 & 2.3 & 3.1 & 3.9 \\
$V_{f}^{*}$ & Net profit from wild fishery & currency & 0.02 & 0.02 & 0.04 & 0.15 & 1.65 \\
$V_{N}^{*}$ & Net profit from aquaculture & currency & 1.6 & 1.8 & 2.3 & 2.9 & 2.3 \\
\hline
\end{tabular}

For an MPA size of $m>0.6$ the model does not give appropriate solutions for our choice of data. We observe from Table 2 that the fishable stock size decreases for an increasing MPA size, while the total stock size declines for small MPA size (i.e., $m \leq 0.3$ ) and increases for a larger MPA compared to the case without an MPA. The wild catch increases for an increasing MPA size and is always larger than the wild catch without an MPA, as long as $m$ is not greater than 0.6 . The total net profits as well as the net profit from the wild fishery increase for an increasing MPA size, while the net profit from aquaculture increases up to $m=0.5$. The size of the aquaculture activity also increases for an increasing MPA size up to $m=0.5$, and declines for a larger MPA, but is always higher than without an MPA.

These numerical simulation results are an illustration of the model, and are a function of the chosen parameters. We therefore examine the effects of small changes (10\% increase) in each parameter value on the optimal solutions without an MPA $\left(X^{*}, Y_{f}^{*}, N^{*}, V^{*}\right)$ and with an MPA $\left(X_{1}^{*}, X_{2}^{*}, Y_{1 f}^{*}, N^{*}, V^{*}\right)$. The model exposes varying degrees of sensitivity to changes in the range of parameters. In order to

\footnotetext{
${ }^{4}$ The stock size, $x_{1}, x_{2}, x$, and catch in weight, $y_{1 f}$, can be calculated as follows:

$x_{1}^{*}=(1-m) K X_{1}^{*} ; x_{2}^{*}=m K X_{2}^{*} ; x^{*}=x_{1}^{*}+x_{2}^{*} ; y_{f}^{*}=K F\left(X^{*}, N^{*}\right)$;

$y_{1 f}^{*}=(1-m) K\left[F\left(X_{1}^{*}, N^{*}\right)+F\left(X_{2}^{*}\right)\right]$
} 
compare these sensitivities, we present these changes as elasticities (ratios of percentage changes in the variable of interest to percentage changes in the parameter values) in the neighborhood of the initial values. The results are presented in Table 3.

Table 3. Elasticities of variables with and without an MPA for a 10\% increase in the parameter values, under optimal management.

\begin{tabular}{|c|r|r|r|r|r|r|r|r|r|}
\hline \multirow{3}{*}{ Parameter } & \multicolumn{9}{|c|}{ Optimal value } \\
\cline { 2 - 10 } & \multicolumn{3}{|c|}{ Without an MPA } & \multicolumn{1}{c|}{ With an MPA $(m=0.3)$} \\
\cline { 2 - 10 } & \multicolumn{1}{|c|}{$X^{*}$} & \multicolumn{1}{|c|}{$Y_{f}^{*}$} & \multicolumn{1}{|c|}{$N^{*}$} & \multicolumn{1}{c|}{$V^{*}$} & \multicolumn{1}{c|}{$X_{1}^{*}$} & \multicolumn{1}{c|}{$X_{2}^{*}$} & \multicolumn{1}{c|}{$Y_{1 f}^{*}$} & \multicolumn{1}{c|}{$N^{*}$} & $V^{*}$ \\
\hline$w$ & 0.05 & 1.70 & - & -0.23 & 0.07 & 0.06 & 1.73 & -0.02 & 0.06 \\
$\alpha$ & -0.05 & -1.00 & -1.10 & -1.14 & -0.03 & -0.03 & -1.13 & -1.09 & -1.11 \\
$v$ & -0.02 & -0.79 & - & 0.08 & -0.03 & -0.02 & -0.83 & 0.02 & -0.01 \\
$c$ & 0.99 & 0.52 & - & 0.48 & 1.00 & 0.88 & 0.79 & -0.08 & 0.50 \\
$q$ & -1.08 & -0.38 & - & -0.56 & -1.09 & -0.96 & -0.63 & 0.08 & -0.51 \\
$p$ & -1.06 & 0.35 & - & -0.69 & -1.06 & -0.93 & 0.08 & 0.06 & -0.54 \\
$r$ & - & - & 1.01 & 1.12 & - & 0.13 & 0.41 & 1.01 & 1.00 \\
$b$ & -0.00 & - & - & -0.00 & -0.00 & -0.00 & - & - & -0.00 \\
$\gamma$ & & & & & - & -0.12 & -0.09 & - & -0.00 \\
\hline
\end{tabular}

The stock size with or without an MPA is more sensitive to changes in the unit price of fish, $p$, and the catchability, $q$, but it is less sensitive to changes in remaining parameter values. The harvest is more sensitive to changes in the marginal productivity of aquaculture, $w$, and the aquaculture sensitivity coefficient, $\alpha$, while the size of aquaculture activity and the total net profits are more sensitive to changes in the aquaculture sensitivity coefficient, $\alpha$ and the intrinsic growth rate, $r$.

We notice that the implementation of an MPA size of $m=0.3$ either increases or decreases the degrees of sensitivity of variables to changes in the parameter values. For instance, we observe that a 10 percent increase in the parameter values increases mainly the sensitivity of the stock size, the wild catch, and the size of the aquaculture activity compared to the situation without an MPA, while the sensitivity of the total discounted profits are mostly reduced. However, the difference between the elasticities of the optimal values with an MPA and without an MPA is very small.

\section{Discussion and Conclusion}

The introduction of an MPA can function as a sustainable management tool in order to rebuild depleted populations of exploited species and safeguard the spawning biomass. Benefits for fisheries from MPAs are expected from egg and larvae dispersal as well as from spillover of adults to adjacent fishing grounds (Goñi et al., 2010). We develop a framework to analyze the tradeoff between a wild fishery and aquaculture occurring in the same region, and where the products are sold in the same 
market, including the influence of an MPA. In the model, aquaculture reduces the intrinsic growth of the wild fish stock, and an MPA is introduced to limit the negative effect of aquaculture on the wild fishery, thereby potentially reducing conflicts between the two sectors. The framework can be used to identify these interactions under both an open access fishery and economic optimal management.

Our results suggest that there are tradeoffs between a wild fishery and aquaculture which occur in the same region and are identical in products and price. In the pure open access, the equilibrium wild stock level is independent of the presence of aquaculture, while the wild catch is affected by aquaculture production. The larger the aquaculture production, the lower the wild catch. Establishing an MPA where wild fishing and aquaculture are prohibited results in the total wild stock increasing and being larger than the pure open access case, while the wild catch varies depending on aquaculture development and the MPA size. Creation of an MPA of a certain size may yield greater harvest than the pure open access case when aquaculture production has a significant effect on the wild fishery. For instance, as the aquaculture operation is expanded (for $N>0.002$ in this simulation) resulting in a reduction of more than $50 \%$ of the intrinsic growth rate of fish then introducing an MPA of a certain size increases the wild catch which can reach a maximum value and be greater than when there is no MPA present. The model also clearly illustrates how an open access fishery may completely eradicated in the presence of aquaculture (see Figure 2c), and how an MPA may alleviate this in the sense that some harvest is allowed, though less than under pure open access. This is reminiscent of the Vietnamese lobster fishery's demise in the presence of aquaculture. In recent years Vietnam has had an ambitious MPA plan, but the MPAs that have been implemented have largely had conservation rather than fisheries management focus.

In the optimal management regime, introducing an MPA of a certain size (i.e., $m>0.3$ here) yields larger total stock size, while a small MPA yields larger wild catch, amount of aquaculture activity, total net profits, and net profit from each operation. These values are higher than when there is no MPA. However, the efficiency of aquaculture production reaches its maximum value for MPA size $m$ $=0.5$ and declines for larger MPA size. MPAs combined with an economically optimal management approach outside the reserve do yield better results than the optimal management without an MPA. This is in line with (Ami et al., 2005) who carried out a theoretical study in order to show that both the economic and biological situations are enhanced by the creation of MPAs. They suggest that protecting areas with higher intrinsic growth rates result in higher stock density, fishing effort, and present value in the fishery sector, compared to when there is no MPAs. Sensitivity analysis of the optimal outcomes with and without an MPA in relation to parameter values in our study shows very limited difference in the elasticities, implying low variance when an MPA is introduced in the model. The addition of an MPA effect has no systematic influence on the results of the model. The model therefore seems to be reasonably robust. However, in the case of Vietnamese lobster fishery, optimal management would presumably require prohibitive control measures. 
In our study, the benefits of an introduced MPA from the perspective of aquaculture or fisheries vary dependent on MPA sizes. The managers therefore must take into account management objectives when choosing an MPA size. This work obviously requires analysis of costs and benefits to extractive users (fishermen and farmers). Aquaculture production can affect the wild fishery either via the carrying capacity of fish, the intrinsic growth rate or both. Using MPAs in management of natural resource user conflicts may give different results for each effect. Comparing the similarities/differences in the optimal management results in each case is something the authors plan to follow up in future work.

\section{Acknowledgments}

We would like to thank Ola Flaaten, Ngoc Quach Thi Khanh and colleagues at the Norwegian College of Fisheries Science for valuable input to earlier versions of the paper. Bui Bich Xuan acknowledges funding from NORAD.

\section{Reference}

Ami, D., Cartigny, P., \& Rapaport, A. (2005). Can marine protected areas enhance both economic and biological situations? Comptes Rendus Biologies, 328(4), 357-366.

Anderson, J. L. (1985). Market interactions between aquaculture and the common property commercial fishery. Cran; Russak.

Armstrong, C. W., \& Reithe, S. (2001). Comment: marine reserves: will they accomplish more with management costs? Marine Resource Economics, 16(2), 165-175.

Asche, F., \& Tveterås, S. (2004). On the relationship between aquaculture and reduction fisheries. Journal of Agricultural Economics, 55(2), 245-265.

Chopin, T., Buschmann, A. H., Halling, C., Troell, M., Kautsky, N., Neori, A., . . Neefus, C. (2001). Integrating seaweeds into marine aquaculture systems: a key toward sustainability. Journal of Phycology, 37(6), 975-986.

Conrad, J. M. (1999). The bioeconomics of marine sanctuaries. Journal of Bioeconomics, 1(2), 205217.

Diana, J. S. (2009). Aquaculture production and biodiversity conservation. Bioscience, 59(1), 27-38.

FAO. (2012). World review of fisheries and aquaculture. www.fao.org/docrep/016/i2727e/i2727e01.pdf

FAO. (2013). Global Aquaculture Production Statistics for the year 2011. $\mathrm{ftp} / / / \mathrm{ftp}$.fao.org/FI/news/GlobalAquacultureProductionStatistics2011.pdf

Fisheries, FAO. (2011). State of world aquaculture 2010. Food and Agriculture Organization of the United Nations. www.fao.org/docrep/014/ba0132e/ba0132e.pdf

Flaaten, O., \& Mjølhus, E. (2010). Nature reserves as a bioeconomic management tool: a simplified modelling approach. Environmental and resource economics, 47(1), 125-148.

Goñi, R., Hilborn, R., Díaz, D., Mallol, S., \& Adlerstein, S. (2010). Net contribution of spillover from a marine reserve to fishery catches. Marine Ecology Progress Series, 400, 233-243.

Grafton, R. Q., Kompas, T., \& Van Ha, P. (2006). The Economic Payoffs from Marine Reserves: Resource Rents in a Stochastic Environment*. Economic Record, 82(259), 469-480.

Grafton, R. Q., Kompas, T., \& Van Ha, P. (2009). Cod today and none tomorrow: The Economic Value of a Marine Reserve. Land Economics, 85(3), 454-469.

Hannesson, R. (1998). Marine reserves: what would they accomplish? Marine Resource Economics, $13,159-170$. 
Hannesson, R. (2003). Aquaculture and fisheries. Marine Policy, 27(2), 169-178.

Hoagland, P., Jin, D., \& Kite-Powell, H. (2003). The optimal allocation of ocean space: aquaculture and wild-harvest fisheries. Marine Resource Economics, 18(2), 129-148.

Naylor, R. L., Goldburg, R. J., Primavera, J. H., Kautsky, N., Beveridge, M. C., Clay, J., . . Troell, M. (2000). Effect of aquaculture on world fish supplies. Nature, 405(6790), 1017-1024.

Petersen, E., Tuan, L., \& Glencross, B. (2011). Bioeconomics of lobster, Panulirus ornatus, culture in Vietnam. ACE Discussion Paper, 5.

Phu, T. D., Nhuan, N. V., Luong, N. T., Thong, P. V., Vang, N. Y., Khanh, N. Q., Chien, T. N. (2013). Đánh giá tác động của nghề khai thác tôm hùm giống đến cảnh quan môi trường và nguồn lợi thuỷ sản vịnh Nha Trang. Đề tài Khoa học Công nghệ. Trường Đại học Nha Trang. (In English: Assessment of the impacts of the juvenile lobster fishery on the landscape, environment, and marine resource in Nha Trang Bay. Science and Technology Research. Nha Trang University.

Sanchirico, J. N., \& Wilen, J. E. (2001). A bioeconomic model of marine reserve creation. Journal of Environmental Economics and Management, 42(3), 257-276.

Simon, M., Fromentin, J. M., Bonhommeau, S., Gaertner, D., Brodziak, J., \& Etienne, M. P. (2012). Effects of stochasticity in early life history on steepness and population growth rate estimates: an illustration on Atlantic bluefin tuna. PLoS One, 7(10), e48583.

Smith, M. D., \& Wilen, J. E. (2003). Economic impacts of marine reserves: the importance of spatial behavior. Journal of Environmental Economics and Management, 46(2), 183-206.

Tacon, A. G., \& Metian, M. (2009). Fishing for aquaculture: non-food use of small pelagic forage fish - a global perspective. Reviews in Fisheries Science, 17(3), 305-317.

Thuy, N.T.B., \& Ngoc, N.B. (2004). Current status and exploitation of wild spiny lobsters in Vietnamese waters. In spiny lobster ecology and exploitation in the South China Sea region. ACIAR Proc, 120, 13-16.

Ye, Y., \& Beddington, J. R. (1996). Bioeconomic interactions between the capture fishery and aquaculture. Marine Resource Economics, 11, 105-124. 